\title{
The Correlation between Personality Dimensions Measured by the Questionnaire Called Velikih $5+2$ and Dimensions of the Level of Aspiration
}

\author{
Rajković Dragica, MA
}

\author{
Department of Psychology, Faculty of Philosophy-Nikšić
}

University of Montenegro

\section{Doi:10.5901/jesr.2014.v4n4p49}

\section{Abstract}

This study is about searching the correlation between personality dimensions and dimensions of the level of aspiration The study applied two instruments: Velikih 5+2 ( VP+2 ; Smederevac, Mitrovic and Čolović , 2009), which assesses seven dimensions of personality and their subscales, which are Neuroticism, Conscientiousness, Pleasantness, Extraversion, Openness to experience, Negative valence and Positive valence, and Aspiration Index (aspiration Index, Kasser and Ryan , 1996 ) designed to measure the level of intrinsic and extrinsic aspiration. The results show correlation between the Positive valence and Importance of extrinsic values, the Probability of intrinsic values, the Probability of extrinsic values and the Achievement of extrinsic values. Negative valence correlates with the Importance of extrinsic values, the Probability of intrinsic values, the Probability of extrinsic values, the Achievement of intrinsic values and the Achievement of extrinsic values. Openness is associated with the Probability of intrinsic values, the Importance of intrinsic values, the Achievement of extrinsic values and the Achievement of intrinsic values. Extraversion is associated with the Importance of intrinsic values, while Conscientiousness correlates with the Achievements of intrinsic values. When it comes to gender, there is a significant correlation gained with variables measuring Importance of intrinsic values and Probability of intrinsic values, and the results show that higher scores on these dimensions are achieved by women.

Keywords: level of aspiration, personality dimensions , multivariate analysis of covariance

\section{Introduction}

The study is about searching the correlation between personality dimensions anf the level of aspiration. Recent research now prove that people reported about less positive and more negative mood, less life satisfaction, poorer psychological adaptation when achievement of intrinsic goals is less important then extrinsic goals. Several correlative studies have demonstrated that people who have strong aspirations for extrinsic life goals, relative to intrinsic ones, show signs of poorer satisfaction od basic needs, lower quality bonds,less cooperative behavior, a lot od prejudice and compliance with the attitude that is currently is dominated in society. This pattern is present in different cultures and in different age groups. (Kasser and Ryan, 1996. )

What are the factors that push toward extrinsic motivation, although it is unlikely that it will lead them to heppiness and well-being?Although the answer to the question is not certainly one-sided, we believe that the selection of targets can become more extrinsic rather rhen intrinsic when people experience psychological threats, which makes them feel insecure or anxious .

Variability of individual differences is almost endless, but most of these differences are considered insignificant, take place every day in interpersonal interactions and on that way remain largely unnoticed. Francis Galton is one of the first scientist who explicity pointed out the importance of lexical hypothesis, whoch says that the most important individual differences in human transactions will be coded in terms of some or all of the languages of the world. As a result of the second one psycho lexical study in the Serbian languare was created questionnaire VP+2, which we use in this study to measure personality traits by it.

The study was conducted in the therritory of Montenegro and Serbia. The sample consisted of 199 subjects, aged 18 to 60 years. Respondents of both sexes and 90 male respondents and 109 female respondents . Level of educational attainment of the respondents ranged from elementary to high school education.

\section{Instruments}

During the investigation thing used the following instruments :

1.Velikih 5+2 ( +2 VP; Smederevac Mitrovic and Čolović, 2009). This questionnaire is the product of second psycholexical study in Serbian language which assesses 7 dimensions of personality and their subscales: 
1. Neuroticism This dimension refers to individual differences in reactivity to potentially threatening stimulant. Subscales in this dimensions are: anxiety, depression, and negative affect

2. Conscientiousness - This is the second dimension of individual differences related to the attitude towards obligations. In this dimension volition is dominating component. Subscales are negligence, self-discipline and respect for rules.

3. Pleasantness -This dimension includes three subscales, namely: Aggression, peace and a temper . It is clear that two of the three dimensions directed towards conflict interpersonal relationships, and the only one in the direction of the pursuit of harmonious even the dimension is called pleasantness, to other dimensions in the context of the results of studies of lexical

4. Extraversion- In this dimension, individual differences in responsiveness to the external environment are assessed. This dimension consists of three subscales, namely: sociability, positive affect and cordiality .

5. Openness to experience this dimension refers to the tendency towards progress and development and largely determined by cultural factors, as manifestations of this tendency in their daily lives must be shaped by culture. Includes subscales search for novelty and intellect .

6. negative valence subscale of this dimension are manipulative and negative self - concept. When it comes to manipulative people who achieve high scores are intrusive, boastful and prone to manipulation. Negative self concept, ie. high score on this scale speaks of expressed negative self-evaluation, which is a consequence of the experience of their incompetence .

7. positive valence This dimension includes subscales: Superiority and positive self concept. People who have overrated opinion of themselves and who want to be close to the centers of power have high scores at the subscale of superiority. When it comes to positive self concept of high scores with people who have high selfesteem and a positive attitude to their own abilities, talents and virtues. These people invest a lot of on themselves and their education and then feel pride and a sense of their qualities .

A five-point scale and a Likert -type ( answer by circling the numbers 1 to 5 , while numbers indicate the following: 1 - strongly disagree , 2- mostly disagree , 3- not sure , 4- mostly agree , 5- strongly agree). The scale consists of 198 items .

2. Index of aspiration ( Aspiration Index , Kasser and Ryan , 1996) The authors of the scale are Kasser and Ryan , and the scale first appeared in an article published in the Journal of Personality and Social Psychology Bulletin. Other scales have fewer items, 35 because they are items that are not shown to be predictive in the first scale ( 42 items ) revised or omitted. From the 35 items included in the aspirations of the category life goal. Categories are: wealth , popularity, image, physical health, personal growth, affiliation and contribution to the community. The three categories are related to extrinsic value ( riches, popularity, image) and four related to intrinsic values ( personal growth, affiliation, physical health, contribution to the community). In addition to the first version of the scale assessed the importance of the goal and the chance to achieve it in the second version are evaluated : 1.probability(likelyhood) 2.importance 3.achievement. In answering using five- or seven-level scale, but the authors believe that it $d$

oes not lead to significant differences in the results obtained. Respondents answer on a five-point of Likert scales .

\section{Data Analysis Method and Results}

Data analysis was performed in the statistical package SPSS for Windows . In the first phase of data analysis, principal components analysis was applied in order to reduce the initial set of variables. The initial set of variables in this study consisted of respondents' answers to the 35 items of the scale aspirations and respond to 198 items of the questionnaire . Within VP +2 responses to items were reduced to the first principal component, ie . seven subscales. In further analysis were used factor scores on the principal components of all seven dimensions - neuroticism, extraversion, agreeableness , conscientiousness and openness to experience, both positive and negative valence. And when it is scale of aspirations factor scores on the principal components of the six dimensions of space questionnaire for the assessment of aspiration were used.

Correlation between gender and personality traits of the area measurement questionnaire Big Five plus two and dimension of the space scales of aspirations were examined by multivariate analysis of covariance. In this analysis, categorical predictor sex, continuous predictors (covariates) are the factor scores on the principal components of the seven scales of the questionnaire Big Five Plus Two, a criterion variables were the factor scores on the six dimensions of space questionnaire for the assessment of aspiration: The first three dimensions are related the extrinsic value (the value , importance and achievements in the domain of the value), and the other three on the intrinsic value ( the value again , the importance and achievement, but this time in the domain of intrinsic value) . 
Table no.1 Multivariate predictors effects

\begin{tabular}{|l|c|c|c|c|c|}
\hline & Wilks Lambda & F & Df(efectt) & Df(error) & P \\
\hline Intercept & 0.999 & 0.018 & 6 & 185 & 1.000 \\
\hline Neuroticism & 0.947 & 1.732 & 6 & 185 & 0.116 \\
\hline Extraversion & 0.970 & 0.962 & 6 & 185 & 0.452 \\
\hline Conscientiousness & 0.948 & 1.705 & 6 & 185 & 0.122 \\
\hline Pleasantness & 0.982 & 0.565 & 6 & 185 & 0.758 \\
\hline Openness to experience & 0.878 & 4.275 & 6 & 185 & 0.000 \\
\hline Positive valence & 0.890 & 3.792 & 6 & 185 & 0.001 \\
\hline Negative valence & 0.906 & 3.203 & 6 & 185 & 0.005 \\
\hline Gender & 0.942 & 1.905 & 6 & 185 & 0.082 \\
\hline
\end{tabular}

When we look at the chart we can coclude that Openness, Positive valence And Negative valence correlate with criteria variables. The multivariate effect of other dimensions is not proved to be significant.

Table no .2 Multiplecorrelation Coefficient

\begin{tabular}{|l|c|c|c|c|c|}
\hline & Correlation coefficient $(\mathrm{R})$ & $\mathrm{R}$ Square $\left(\mathrm{R}^{2}\right)$ & Adjusted R Square $\left(\mathrm{R}^{2}\right)$ & $\mathrm{F}$ & $\mathrm{p}$ \\
\hline The importance of extrinsic value & 0.494 & 0.244 & 0.212 & 7.662 & 0.000 \\
\hline The importance of intrinsic value & 0.557 & 0.311 & 0.282 & 10.703 & 0.000 \\
\hline The probability of extrinsic value & 0.479 & 0.229 & 0.197 & 7.068 & 0.000 \\
\hline The probability of intrinsic value & 0.589 & 0.347 & 0.319 & 12.608 & 0.000 \\
\hline The attainment of extrinsic value & 0.482 & 0.233 & 0.200 & 7.203 & 0.000 \\
\hline The attainment of intrinsic value & 0.565 & 0.319 & 0.290 & 11.131 & 0.000 \\
\hline
\end{tabular}

The coefficient of multiple correlations were significant for all predictors and their values indicate a moderate correlation. Therefore, in this study, all criteria were significantly associated with a set of predictors, where the connection with variable intrinsic probability of maximum intensity.

Positive valence was significantly associated with the importance of extrinsic value $(\beta=0.43, p<0.001)$, the probability of intrinsic value $(\beta=0.20, p<0.05)$, the probability of extrinsic value $(\beta=0.31, p<0,001)$, the achievements of extrinsic value $(\beta=0.23, p<0.05)$. All beta coefficients have a positive sign. Negative valence associated with the importance of extrinsic value $(\beta=0.22, p<0.05)$, the probability of intrinsic value $(\beta=0.32, p<0.001)$, the probability of extrinsic value $(\beta=0.17, p<0,05)$, the attainment of intrinsic value $(\beta=0.36, p<0.001)$, the attainment of extrinsic value $(\beta=0.27, p<0.05)$. Openness is associated with the likelihood of intrinsic value $(\beta=0.29, p<0.001)$, the importance of intrinsic value $(\beta=0.33, p<0.001)$, the achievements of extrinsic value $(\beta=0.21, p<0.05)$ and achievements of the intrinsic value $(\beta=0.26, p<0.05)$. Other predictors are connected by a plurality of dependent variables. Extraversion is associated with the importance of intrinsic value $(\beta=0.22, p<0.05)$, and Conscientiousness with the achievements of intrinsic value $(\beta=0.23, p<0.05)$. All beta coefficients have a positive sign when it comes to sex significant correlation was obtained with the variable importance of intrinsic value $(\beta=-0.16, p<0.05)$ and the probability of intrinsic value $(\beta=$ $0.12, p<0,05)$, and the results indicate that the higher the scores on the dimensions of the women achieved.

Table no.3 Parcial contribution of predictors

\begin{tabular}{|l|c|c|c|c|c|c|}
\hline & $\begin{array}{c}\text { Importance of } \\
\text { extrinsic value }\end{array}$ & $\begin{array}{c}\text { Importance of } \\
\text { intrinsic value }\end{array}$ & $\begin{array}{c}\text { The probability of } \\
\text { extrinsic value }\end{array}$ & $\begin{array}{c}\text { The probability } \\
\text { of intrinsic value }\end{array}$ & $\begin{array}{c}\text { The attainment of } \\
\text { extrinsic value }\end{array}$ & $\begin{array}{c}\text { The attainment } \\
\text { of intrinsic value }\end{array}$ \\
\hline & $\mathrm{B}$ & $\mathrm{B}$ & $\beta$ & $\mathrm{B}$ & $\mathrm{B}$ & $\beta$ \\
\hline Intercept & 0.075 & -0.049 & -0.088 & -0.133 & -0.051 & -0.134 \\
\hline Neuroticism & 0.036 & $\mathbf{0 . 2 2 3 ^ { * }}$ & 0.063 & 0.104 & 0.036 & 0.077 \\
\hline Extraversion & 0.001 & -0.084 & 0.058 & 0.102 & 0.157 & $\mathbf{0 . 2 2 9 ^ { * * }}$ \\
\hline Conscientiousness & -0.067 & -0.062 & -0.050 & -0.011 & -0.052 & 0.050 \\
\hline Pleasantness & 0.063 & $\mathbf{0 . 3 3 2 ^ { * * }}$ & 0.149 & $\mathbf{0 . 2 9 2}^{* *}$ & $0.211^{*}$ & $\mathbf{0 . 2 6 2 ^ { * * }}$ \\
\hline $\begin{array}{l}\text { Openness to } \\
\text { experience }\end{array}$ & $\mathbf{0 . 4 2 8 ^ { * * }}$ & 0.107 & $\mathbf{0 . 3 0 7 ^ { * * }}$ & $\mathbf{0 . 2 0 3 ^ { * }}$ & $\mathbf{0 . 2 3 4 ^ { * }}$ & 0.156 \\
\hline Positive valence & $\mathbf{0 . 2 2 5 ^ { * }}$ & $\mathbf{0 . 0 5 3}$ & $\mathbf{0 . 3 2 2 ^ { * * }}$ & $\mathbf{0 . 1 6 9 ^ { * }}$ & $\mathbf{0 . 3 5 8 ^ { * * }}$ & $\mathbf{0 . 2 7 1 ^ { * * }}$ \\
\hline Negative valence & -0.062 & $-\mathbf{0 . 1 5 8 ^ { * }}$ & -0.052 & $-\mathbf{0 . 1 2 3 ^ { * }}$ & -0.080 & -0.078 \\
\hline Gender & & & & & & \\
\hline
\end{tabular}


These results are indicative of the relationship between gender, personality traits and dimensions of the scale aspirations. It is not proven the existence of a significant correlations of Pleasure and Neuroticism with any of the dimensions of the level of aspiration. Conscientiousness and Extraversion were significantly correlated with one dimension related to intrinsic value, while the remaining three dimensions of space questionnaires Big Five plus two more dimensions correlated with the level of aspiration.

\section{Conclusions}

The results show the correlations (connection) between Extraversion and the Importance of intrinsic goals. We can suppose that the cause of this correlation sub-dimensions of Cordiality and Sociability and that achievement of goals related to the contribution of the community is particularly important to people with these characteristics and what is one of the categories of intrinsic motivation. Of course this does not exclude the correlation of achievement in the field of personal development, affiliation and physical health with extraversion, moreover, we can say that the achievement of intrinsic goals is important for extraverts to establish a quality contact with other people and managed to adequately respond to the demands of the environment .

There is an evident connection between Conscientiousness and the achievements of intrinsic value. A high score on Conscientiousness dimension is typical for people who are ambitious, and want to work and invest in themselves in order to achieve their goals. We can say that it is quite natural that, consequently, they realized people, both in work and private life. Usually, the achievement of these individuals higher than average, and are in some way motivated to be more persistent, more durable and more valuable. Therefore it is reasonable to expect a satisfactory achievements in the field of personal development and contribution to the community and therefore the field of intrinsic value.

Openness to experience shows correlation with all three dimensions of intrinsic value, and achievement of extrinsic value. This feature includes the ability to improve and progress in intellectual terms and in terms of experiencing new experiences. It can also refer to personal development ( regarding intellect and physical health ) and to contribute to the community. These people are largely devoted to themselves and want to improve (increase) their knowledge and abilities, therefore the achievement of some intrinsic values is also essential, as well as constantly working on it to give some results and there is satisfaction with that that has been achieved in the realization of extrinsic goals. Work on achieving intrinsic goals means that a person will acquire new knowledge, skills and experiences, the development of communication society and strengthening links with the individuals in it, as well as the contribution of the very community in which one lives. All these papers attract people who have a high score on Openness to experience, and therefore choose this type of motivation as a default.

The positive valency is associated with all three dimensions of extrinsic values and with of probability of achieving intrinsic value. According to the fact that people with high scores on this dimension consider themselves superior to others is not surprising that they find that they have reached a higher level of achievement of certain goals, as well as the high probability that these goals will be fully realized in the future. Inclination of the people towards positive selfdescription probably was not missing either during this test, and therefore we have these results. The results show that special attention is paid to extrinsic values. We can say that the wealth, image and popularity is very important to these people because it helps them feel powerful and superior to others. Because of the fact that people characterized by a positive self concept is always trying to get positive feedback (which motivates them to progress ), popularity in society and recognizable image represent a way to achieve that goal. Recognizable image and popularity in social circles make them be in the center of attention and people perceive them as special and different from others, and they have need for that. It Makes them feel more important than others, build a positive self-image because it is obvious that other people love them therefore only logical thing is that they love themselves. Material wealth brings a sense of security and power , and besides the image and popularity will contribute such persons to feel superior to other people and to affirm themselves and because of that the achievement of extrinsic goals is very important. However, those people characterized by high scores on the Positive valence believe that the achievement of intrinsic goals is important, ie . they believe in the possibility that they will achieve them in the future. This comes from their belief in themselves and their abilities of awareness of one's own qualities, but also from an idea that is in base of their attitude of superiority to others, and that is the idea that they are destined for great things. From this idea, thought that they can achieve anything they want, and their achievement of intrinsic goals in the future will not be a problem was probably born.

Negative valence does not correlate only with the importance of achieving intrinsic value. As the negative valence dimension which is characterized by self -presenting in a bad light, and low attitude about themselves, it seems that perhaps these people's desire to become better and worthy of others' attention caused an increased desire to achieve both extrinsic as well as intrinsic value. These people probably perceive the achievement of extrinsic goals as a way to 
begin to respect themselves and to respect others. Wealth , image and popularity seem like a quick solution that will help them people appreciate others to appreciate themselves. However, due to the extremely negative opinions of themselves, these people think they do not deserve a positive opinion of others and are often unable to recognize that if it exists, and probably even the achievement of these goals would not lead to the desired state. Absense of correlation between the importance of achieving intrinsic value could be connected with fact that confidence in the people is shaken at people with high scores on this dimension, (undermined) and there is generally a negative attitude towards them and they think that the achievement of intrinsic goals is not important because it is not appreciated enough in today's society. The fact that these people believe that there is probability that they will achieve these goals in future and there is satisfaction in the accomplishment of the present moment can be explained by the sub-dimension- manipulation. So these people are prone to manipulation and showing off and trying to always express these qualities. They tend to show that they are more capable than others and if that in most situations it is not true . It is possible that these traits come to the fore in patients and during testing, and therefore their scores on the dimensions that measure achievement and the probability of achieving intrinsic and extrinsic values are high.

Regarding sex (gender) women have greater achievement in sizes of intrinsic value importance and probability of intrinsic values. These results show that women pay more attention to affiliation, communicating with other people, and that they are more empathetic, need to help others and contribute to the community. I would say that the need for achievement intrinsic goals is not as important to women as the fact that they are more willing to report about it . It seems that women are much more willing to talk about compassion for others, the achievement of significant intimate interpersonal relationships, personal development, than men and that's why we get such results .

Taking into account the results of previous studies, which show that people who choose extrinsic goals versus intrinsic goals are dissatisfied with themselves and their lives, we can say that in this study these claims are, in some way, re-proven. The fact that the negative valence dimension is not only related to the importance of achieving intrinsic values confirms the existence of negative self-evaluation, lower satisfaction with oneself and one's life at people to whom the achievement of extrinsic goals is very important. Also, anxiety, low self-esteem, insecurity may be the result of some psychological threat and cause marking the extrinsic goals as important (which has already been shown in some studies), and in humans these characteristics lead to high scores on the already mentioned negative valence dimension.

As one of four mini SDT theories, the theory of cognitive evaluation is about the impact of social context on intrinsic motivation, we can explain correlation extraversion dimensions of and openness to experience through this theory. In fact, it is obvious that people who have better social life, pay great attention to social contacts and are always open to new knowledge and experience of more importance to intrinsic goals. People who want to have good relations with others , to be educated, knowledgeable in cultural events, to experience new things, are aware that they will achieve their goals if they want to work on their personal development or if they contribute to the community and so on . So we can say that the results repeated in favor of this choice of extrinsic goals negatively correlated with self-actualization , vitality , global functioning, social productivity, positive affect and life satisfaction .

In this study the assumption of the existence of personality traits and levels of aspiration as a separate category is confirmed, but their strong connection, and the connection and intersection of model of the big five plus two and level of aspirations are emphasized. The obtained results show how personality traits affect the type of life goals that we choose as the most essential.

Due to the fact that in earlier studies correlation of psychological threats with extrinsic goals is repeatedly proven, and it is considered that people primarily meet basic needs, and then it comes the self-actualization, I suggest to include satisfaction with economic status, as one of the variables, in the following research. If some people are not financially provided and their existence is threatened, it is quite possible that due to that, they have primary extrinsic motivation, and that it affected the results of this study. Also I suggest to use more representative samples in the implementation of the following research.

\section{References}

Čolović, P., Mitrović, D., Smederevac, S. (2005). Evaluacija pet velikih u našoj kulturi primenom upitnika FIBI. Psihologija, 38, 55-76.

Kasser, T., \& Ryan, R. M. (1993). A dark side of the American dream: Correlates of financial succes as a central life aspiration. Journal of Personality and Social Psichology, 65, 410-422.

Kasser, T., \& Ryan, R. M. (1996). Further examining the American dream: Differential correlates of intrinsic and extrinsic goals. Personality and Social Psichology Bulletin, 22, 80-87.

Majstorović, N.,(2008) Primena teorije Self-determinacije u istraživanjima radne, Primenjena psihologija, 2008, Vol. 1 (1-2), str. 5-18

Roberts, B.W., O'Donnell, M., \& Robins, R. (2004). Goal and Personality Trait Development in Emerging Adulthood. Journal of Personality and Social Psichology, 87 (4), 541-550. 
Ryan, R. M., Chirkov, V. I., Little, T.D., Sheldon, K.M., Timoshina, E., \& Deci, E. L. (1999). The American dream in Russia: Extrinsic aspirations and well-being in two cultures. Personality and Social Psichology Bulletin, 25.

Ryan, R. M., \& Deci, E. L. (2000). Self-determination theory and the facilitation of intrinsic motivation, social development, and wellbeing. American Psychologist, 55, 68-78.

Sheldon, K. M., \&Kasser, T. (2008). Psychological threat and extrinsic goal striving. Motivation and Emotion, 32, 37-45.

Smederevac, S. i Mitrović, D. (2006). Ličnost-metodi i modeli, Beograd: Centar za primenjenu psihologiju

Smederevac, S., Mitrović, D., i Čolović, P. (2009). Prikaz upitnika Velikih pet plus dva. Nepublikovan materijal. Filozofski fakultet, Univerzitet u Novom Sadu, Novi Sad. 